\title{
The Subunit Structure of Normal
}

\section{and Hemophilic Factor VIII}

\author{
Gabriel A. Shapiro, Judith C. Andersen, Salvatore V. Pizzo, and \\ Patrick A. McKee \\ From the Department of Medicine, Veterans Administration Hospital and the \\ Department of Biochemistry, Duke University Medical Center, \\ Durham, North Carolina 27710
}

\begin{abstract}
A в S T R A C T Human factor VIII from normals and hemophiliacs was partially purified by ethanol and polyethylene glycol precipitations. Final purification was achieved by gel filtration on 2 or $4 \%$ agarose or ion exchange chromatography on diethylaminoethyl cellulose. Comparable amounts of highly purified protein were obtained from normal and hemophilic plasma following the agarose chromatography step. Highly purified factor VIII was not dissociated by 6 M guanidine hydrochloride or $1 \%$ sodium dodecyl sulfate. However, when reduced by $\beta$-mercaptoethanol and analyzed by sodium dodecyl sulfate polyacrylamide gel electrophoresis, a single subunit species with an estimated 195,000 molecular weight was found for both normal and hemophilic factor VIII. By sedimentation equilibrium analysis, the normal factor VIII subunit was homogeneous and had an estimated molecular weight of 202,000. The subunit polypeptides from normal or hemophilic factor VIII contained carbohydrate. Each was homogeneous by isoelectric focusing. Immunodiffusion of purified normal and hemophilic factor VIII against rabbit antiserum to purified normal human factor VIII showed a single line of precipitation. Very low concentrations of purified human thrombin initially increased the activity of normal factor VIII about threefold and then progressively destroyed activity by $3 \mathrm{~h}$. Only minimal activation occurred with hemophilic factor VIII. Both the activation and inactivation of normal and hemo-
\end{abstract}

A preliminary report of this work was given at the 28th meeting of the American Federation for Clinical Research, Chicago, Ill., 1970.

Dr. Shapiro is presently a Hematology Fellow, Department of Medicine, Parkland Hospital, Dallas, Texas. Dr. Andersen is a postdoctoral Fellow of the National Hemophilia Foundation.

Reccived for publication 22 January 1973 and in recised form 16 April 1973. philic factor VIII were unaccompanied by detectable changes in subunit molecular weight. These findings may have implications for the definition of the molecular defect in hemophilic factor VIII.

\section{INTRODUCTION}

Several investigators have shown that highly purified human factor VIII (antihemophilic factor; $\mathrm{AHF}^{1}$ ) can be eluted near the void volume when factor VIII concentrates are filtered through 2 or $4 \%$ agarose columns (1-11). These studies suggested that factor VIII has a molecular weight of approximately 2 million, but aggregation as a possible explanation for this high molecular weight cannot be entirely excluded. It has been shown, however, that even in dissociating solvents factor VIII has a molecular weight at least in excess of $400,000(5)$ and an estimated $s_{25, w}$ value of $16.3(8)$. Human factor VIII is known to be a glycoprotein. containing about 5-10\% carbohydrate (8). Recently it has been suggested that bovine factor VIII is likewise a glycoprotein with a molecular weight in excess of one million and is composed of disulfide linked subunits, the smallest of which may be about 85,000 in molecular weight (10). While some of the physicochemical properties of human factor VIII have been determined $(2-4,8-11)$, the study of its subunit structure has only been recently approached $(5,10-12)$. Particularly in light of the immunological evidence that hemophilic plasma contains nonfunctional factor VIII in quantities comparable to those of functional factor VIII in normal plasma (13-18), the characterization of the structures

${ }^{1}$ Abbreviations used in this paper: AHF, antihemophilic factor; MW, molecular weight; $s_{25, w}$, sedimentation coefficient; SDS, sodium dodecyl sulfate; $\overline{\mathbf{v}}$, partial specific volume. 
of normal and nonfunctional factor VIII could be useful in defining the molecular defect in hemophilia.

We now wish to report the results of studies which show that normal human factor VIII is composed of an undetermined number of large subunits which are homogeneous by size and electrical charge. Normal factor VIII was also compared with hemophilic factor VIII with respect to subunit molecular weight, carbohydrate staining properties, and isoelectric focusing mobility. In addition, the effect of thrombin on the structure of both normal and hemophilic factor VIII has been examined.

\section{METHODS}

Factor VIII assay. Factor VIII activity was measured by two methods: $(a)$ the one-stage partial thromboplastin time according to the technique of Langdell, Wagner, and Brinkhous (19), except that the hemophilic substrate plasma was "activated" by adding $5 \mathrm{mg}$ kaolin (J. T. Baker Chemical Co., Phillipsburg, N. J.) to each $\mathrm{ml}$ of hemophilic substrate plasma and then incubating for $5 \mathrm{~min}$ with stirring at $37^{\circ} \mathrm{C}$; Thrombofax (Ortho Laboratories, Raritan, N. J.) served as the source of cephalin in this assay; and $(b)$ a modified thromboplastin generation test as described by Newman, Johnson, Karpatkin, and Puszkin (20). Factor VIII elution patterns during gel chromatography were monitored by determining clotting times for undiluted samples in the one-stage kaolin-activated partial thromboplastin time. Although the eluting buffer had an ionic strength of 0.3 , the final ionic strength of the clotting assay mixture was 0.155 ; final ionic strengths ranging from 0.13 to 0.17 did not affect the clotting times of the assay mixtures. The reference plasma used in these assays was lyophilized pooled plasma standardized with respect to factor VIII content (courtesy of Dr. D. L. Aronson, Division of Biologics Standards, National Institutes of Health) and a freshfrozen house standard plasma, repeatedly assayed against the reference plasma. Units of factor VIII activity were calculated by defining $1 \mathrm{U}$ of factor VIII as that amount contained in $1 \mathrm{ml}$ of normal male plasma (21)

Protein concentrations. These were estimated by the absorbance at $280 \mathrm{~nm}$ or by the method of Waddell (22). Where more quantitative measurements were indicated, the method of Lowry, Rosebrough, Farr, and Randall (23) was used.

Partial purification of normal factor VIII. The starting material in these experiments was either fresh frozen plasma, cryoprecipitate or glycine-precipitated factor VIII (Method IV, Hyland Div., Travenol Laboratories, Costa Mesa, Calif.). In each case, partially purified factor VIII was prepared by the method of Newman et al. (20) modifications being: that glycine-precipitated factor VIII concentrates were not subjected to the $3 \%$ ethanol precipitation step, but were introduced into the purification scheme at the $0.05 \mathrm{M}$ Tris hydrochloride extraction step; and that a bentonite adsorption step was utilized to decrease fibrinogen content prior to precipitation with polyethylene glycol. This latter step was performed by adding bentonite (Sigma Chemical Co., St. Louis, Mo.) to a final concentration of $10 \mathrm{mg} / \mathrm{ml}$ to the "intermediate purity" factor VIII of Newman et al. (20). This mixture was incubated at room temperature and stirred slowly for $5 \mathrm{~min}$ and then centrifuged in polycarbonate bottles at $6,000 \mathrm{~g}$ for $15 \mathrm{~min}$ at $20^{\circ} \mathrm{C}$. The remaining precipitation steps as described by Newman et al. (20) were then carried out on the supernatant fluid. The final precipitate was dissolved in $0.30 \mathrm{M}$ sodium chloride$0.05 \mathrm{M}$ Tris hydrochloride, $\mathrm{pH} 7.4$ to a final protein concentration of $30 \mathrm{mg} / \mathrm{ml}$. This partially purified factor VIII will henceforth be called Step 1 factor VIII.

Gel filtration. Gel chromatography was performed at $4^{\circ} \mathrm{C}$ on both 2 and $4 \%$ agarose columns (Sepharose 2B and $4 \mathrm{~B}$, Pharmacia Fine Chemicals, Inc., Piscataway, N. J.). A siliconized glass column, $5 \times 92 \mathrm{~cm}$, was used in these experiments. The void volume was estimated by filtration of an appropriate volume of $0.005 \%$ Blue Dextran 2000 (Pharmacia Fine Chemicals) in $4 \%$ sucrose. The volume of the partially purified factor VIII applied to the column was always $2-3 \%$ of the column volume. Elution was performed with $0.05 \mathrm{M}$ Tris hydrochloride- $0.3 \mathrm{M}$ sodium chloride buffer, $\mathrm{pH} 7.4$, at a pressure of approximately $40 \mathrm{~cm}$. The flow rate averaged $18 \mathrm{ml} / \mathrm{h}$ and $4.2-\mathrm{ml}$ fractions were collected in siliconized glass tubes. The factor VIII eluted from the agarose column will be referred to as Step 2 factor VIII. Each eluant fraction was assayed immediately for factor VIII activity and its absorbance at $280 \mathrm{~nm}$ determined. As noted by Ratnoff, Kass, and Lang, the recovery of factor VIII from the agarose column improved with use (4).

$D E A E$ chromatography. Step 1 normal factor VIII was dissolved in and dialyzed against the starting buffer, $0.05 \mathrm{M}$ Tris, $\mathrm{pH}$ 7.35. Approximately $24 \mathrm{mg}$ of Step 1 normal factor VIII were applied to a $0.9 \times 10 \mathrm{~cm}$ DEAEcellulose column equilibrated with the starting buffer. The column was developed with a $150 \mathrm{ml}$ linear gradient, the limit buffer being $0.05 \mathrm{M}$ Tris- $0.50 \mathrm{M}$ sodium chloride, $\mathrm{pH}$ 7.35. A peak of factor VIII activity was eluted reproducibly at $0.15-0.17 \mathrm{M}$ sodium chloride; this material will be referred to as Step 2-DEAE factor VIII. Selected fractions containing factor VIII activity were pooled, dialyzed against water to remove salt and lyophilized prior to electrophoretic analysis.

Electrophoresis. Polyacrylamide gel electrophoresis in sodium dodecyl sulfate (SDS) was carried out essentially as described earlier (24-26). Approximately $100 \mu \mathrm{g}$ of lyophilized Step 2 factor VIII from each gel chromatography experiment or Step 2-DEAE normal factor VIII was dissolved in $0.10 \mathrm{ml}$ of $1 \%$ SDS-5 $\mathrm{M}$ urea in 0.01 sodium phosphate buffer, $\mathrm{pH} 7.1$, and one-half of this solution was reduced with a final concentration of $0.1 \mathrm{M} \beta$ mercaptoethanol. Each of the non-reduced and reduced samples were then incubated in the $1 \%$ SDS-5 $\mathrm{M}$ urea and $1 \%$ SDS-5 $\mathrm{M}$ urea-0.1 M $\beta$-mercaptoethanol solvents, respectively, at $37^{\circ} \mathrm{C}$ for $12-24 \mathrm{~h}$ following which the entire volume of each was applied to separate, single electrophoretic gels. In most of these studies, $5 \%$ polyacrylamide gels in the presence of $0.1 \%$ SDS were prepared in $15 \times 0.6$ $\mathrm{cm}$ glass columns. Alternatively, glass columns $10 \mathrm{~cm}$ in length were used. The electrophoretic buffer consisted of $0.1 \% \mathrm{SDS}$ in $0.1 \mathrm{M}$ sodium phosphate buffer, $\mathrm{pH}$ 7.1. All electrophoreses were performed at room temperature using a constant current of $6 \mathrm{~mA}$ per gel for about $6 \mathrm{~h}$ at which time the bromophenol blue dye marker $(0.05 \%)$ had migrated within $1-2 \mathrm{~cm}$ of the gel bottom. Following exposure to thrombin, the factor VIII subunit was examined for changes in molecular weight by the high resolution $3.36 \%$ polyacrylamide gel-SDS method (27) as well as on $5 \%$ polyacrylamide gels in SDS. Gels were stained for protein with freshly prepared Coomassie brilliant blue (Colab Laboratories, Inc., Glenwood, IIl.) and were de- 
stained as previously described (25). The subunit molecular weights of factor VIII were calculated from their electrophoretic mobilities and the experimentally determined mobilities of proteins of known molecular weight on $5 \%$ SDS-gels. The following proteins were reduced by $\beta$-mercaptoethanol and used as standards (the assumed molecular weights are given in parentheses; references are given for those molecular weights about which some controversy exists): rabbit muscle myosin $(194,000)(28), \beta$-galactosidase $(120,000)(29)$, phosphorylase A $(92,000)$, human transferrin $(77,000)(30)$, bovine serum albumin $(69,000)$, catalase $(60,000)$, ovalbumin $(43,000)$, pepsin $(35,000)$, and chymotrypsinogen $(26,000)$. Following electrophoresis, certain of the gels containing highly purified normal or hemophilic factor VIII were stained for carbohydrate-containing proteins with the periodic acid-Schiff reagent (31). This method was modified slightly in that after staining, the gels were washed overnight in distilled water and then destained in $50 \%$ methanol for $8 \mathrm{~h}$. The gels were allowed to re-swell in $7 \%$ acetic acid and stored in that solution.

Amino acid composition. Duplicate samples of Step 2 normal factor VIII were hydrolyzed in $6 \mathrm{M} \mathrm{HCl}$ at $110^{\circ} \mathrm{C}$, in z'acuo, for $24,48,72$, and $96 \mathrm{~h}$. The hydrolysates were dried by rotatory evaporation in iacuo, dissolved in distilled water to give a final concentration of $0.25 \mathrm{mg} / \mathrm{ml}$, and then analyzed quantitatively using a Beckman 120B amino acid analyzer (Beckman Instruments, Inc., Fullerton, Calif.) equipped with high sensitivity cuvettes. Half-cystine and methionine contents were determined after oxidation by performic acid to cysteic acid and methionine sulphone, respectively (32). Valine and isoleucine were quantitated from the analysis of 120 -h hydrolysates. Tryptophan was not determined.

Sedimentation equilibrium analysis. A Beckman-Spinco Model E analytical ultracentrifuge was used in these studies. For the determination of subunit molecular weight, the high speed method of Yphantis (33) was used. Purified normal factor VIII was dissolved in 5.6 M guanidine hydrochloride-0.1 M $\beta$-mercaptoethanol to give a protein concentration of approximately $0.30 \mathrm{mg} / \mathrm{ml}$. The speed employed was $20,000 \mathrm{rpm}$, the column height was $2.5 \mathrm{~mm}$, and the temperature was $25 \pm 1{ }^{\circ} \mathrm{C}$. The subunit molecular weight was estimated by using a partial specific volume $(\bar{v})$ of 0.722 which was calculated from the amino acid composition (34) as well as at a $\bar{v}$ value of 0.712 which is the calculated $\overline{\mathrm{v}}$ after correction for preferential binding of guanidine hydrochloride (35). Because of the difficulty in judging the exact value for $\bar{v}$, the subunit molecular weight of human factor VIII was also calculated at $\bar{v}$ values of 0.71 and 0.73 . Other operations in these analyses have been described (36, 37).

Purification of factor VIII from normal and hemophilic plasmas. Following preliminary characterization of normal factor VIII, a series of experiments were performed to compare individual samples of factor VIII prepared from several normal plasmas and factor VIII-like material prepared from several hemophilic plasmas. Venous blood was obtained from each of six normal male volunteers and six hemophiliacs with factor VIII levels $80-120 \%$ and $<1-5 \%$ of normal, respectively, as measured by the method of Langdell et al. (19). Each $360 \mathrm{ml}$ blood sample was collected into $40 \mathrm{ml}$ of cold $3.5 \%$ sodium citrate and immediately centrifuged at $5,000 \mathrm{~g}$ at $4^{\circ} \mathrm{C}$ for $15 \mathrm{~min}$. The supernatant plasma was siphoned from each specimen into separate plastic containers and frozen in a dry ice-ethanol bath. The samples were stored at $-20^{\circ} \mathrm{C}$ and the modified method for the partial purification of factor VIII was carried out on each sample at a convenient time, ranging from 1 to 21 days after collection. Each of the final precipitates resulting from the partial purification of factor VIII was dissolved in $0.30 \mathrm{M}$ sodium chloride- $0.05 \mathrm{M}$ Tris hydrochloride buffer, $\mathrm{pH} 7.4$ to give an average protein concentration of $27.5 \pm 2.8 \mathrm{mg} / \mathrm{ml}$. The individual samples of Step 1 factor VIII from each normal or hemophilic plasma were then applied to a $4 \%$ agarose column $(0.9 \times 56 \mathrm{~cm}$, siliconized) and eluted at $4^{\circ} \mathrm{C}$ with a pressure head of approximately $40 \mathrm{~cm}$, using the $0.30 \mathrm{M}$ sodium chloricle$0.05 \mathrm{M}$ Tris hydrochloride, $\mathrm{pH} 7.4$, buffer. In each case, the sample volume applied to the column was $0.9 \mathrm{ml}$, and $0.9 \mathrm{ml}$ fractions were collected into siliconized glass tubes. Fractions were assayed for factor VIII activity using the one-stage method and protein concentration estimated from the absorbance at $280 \mathrm{~nm}$.

In the case of each normal sample, the eluant fractions which contained the void volume protein peak with factor VIII activity were pooled and dialyzed at $4{ }^{\circ} \mathrm{C}$ against four changes of distilled water in a ratio of 1 part sample to 2,000 parts water. The samples were then quick-frozen and lyophilized in siliconized, conical $15 \mathrm{ml}$ centrifuge tubes. The protein peak in each of the hemophilic elution patterns which corresponded to the active peak in the normal elution patterns was isolated likewise. Each sample was then prepared for analysis by SDS-gel electrophoresis with and without $\beta$-mercaptoethanol as outlined under Electrophoresis.

Isoelectric focusing. Highly purified normal and hemophilic factor VIII preparations were reduced and carboxymethylated according to the method of Hirs (38) except that $6 \mathrm{M}$ guanidine hydrochloride in $0.55 \mathrm{M}$ Tris $\mathrm{HCl}$ buffer, $\mathrm{pH}$ 8.1, containing $0.5 \mathrm{M}$ EDTA was used as the solvent. Following dialysis against distilled water, the samples were concentrated by lyophilization and then analyzed by isoelectric focusing on polyacrylamide gels by the method of Hayes and Wellner (39), modified by reducing the riboflavin concentration from 0.0015 to $0.0005 \%$ since polymerization did not occur at the higher concentration.

Immunology. To insure that the activity of purified fractions was due to the specific presence of factor VIII, activity was determined before and after incubation with plasma from a hemophiliac with a known specific factor VIII inhibitor titer of $1: 512$ as determined by using the partial thromboplastin time test (40). To demonstrate specific inhibition, $0.2 \mathrm{ml}$ of inhibitor plasma was mixed with $0.2 \mathrm{ml}$ of the most active void volume fractions eluted from the gel columns. Following incubation for $30 \mathrm{~min}$ at $37^{\circ} \mathrm{C}$, $0.1 \mathrm{ml}$ of this mixture was assayed by the modified onestage partial thromboplastin time, using hemophilic plasma as substrate. In addition antiserum to normal human factor VIII was prepared by injecting an emulsion containing the most concentrated portion of the $4 \%$ agarose void volume protein (usually $200-300 \mu \mathrm{g}$ in $2-3 \mathrm{ml}$ Tris saline buffer) mixed with an equal volume of alumina $\mathrm{C} \gamma$-Gel (A grade, Calbiochem, Los Angeles, Calif.) into the foot pads of rabbits. This was repeated in $3 \mathrm{wk}$, and 7 days after the final injection $20-30 \mathrm{cc}$ of blood was drawn from an ear artery or by intracardiac puncture. The blood was allowed to clot in glass tubes at $37^{\circ} \mathrm{C}$ for at least $2 \mathrm{~h}$, then centrifuged at $1,500 \mathrm{~g}$ for $20 \mathrm{~min}$. Antiserum was stored in $1 \mathrm{ml}$ aliquots at $-15^{\circ} \mathrm{C}$. Absorbed antiserum was prepared as described by Zimmerman, Ratnoff, and Powell (16) by using the supernatant fluid from the $3 \%$ ethanol cyoprecipitate as the absorbant. Step 2 normal and hemophilic factor VIII was prepared for immunodiffusion by concentrating 

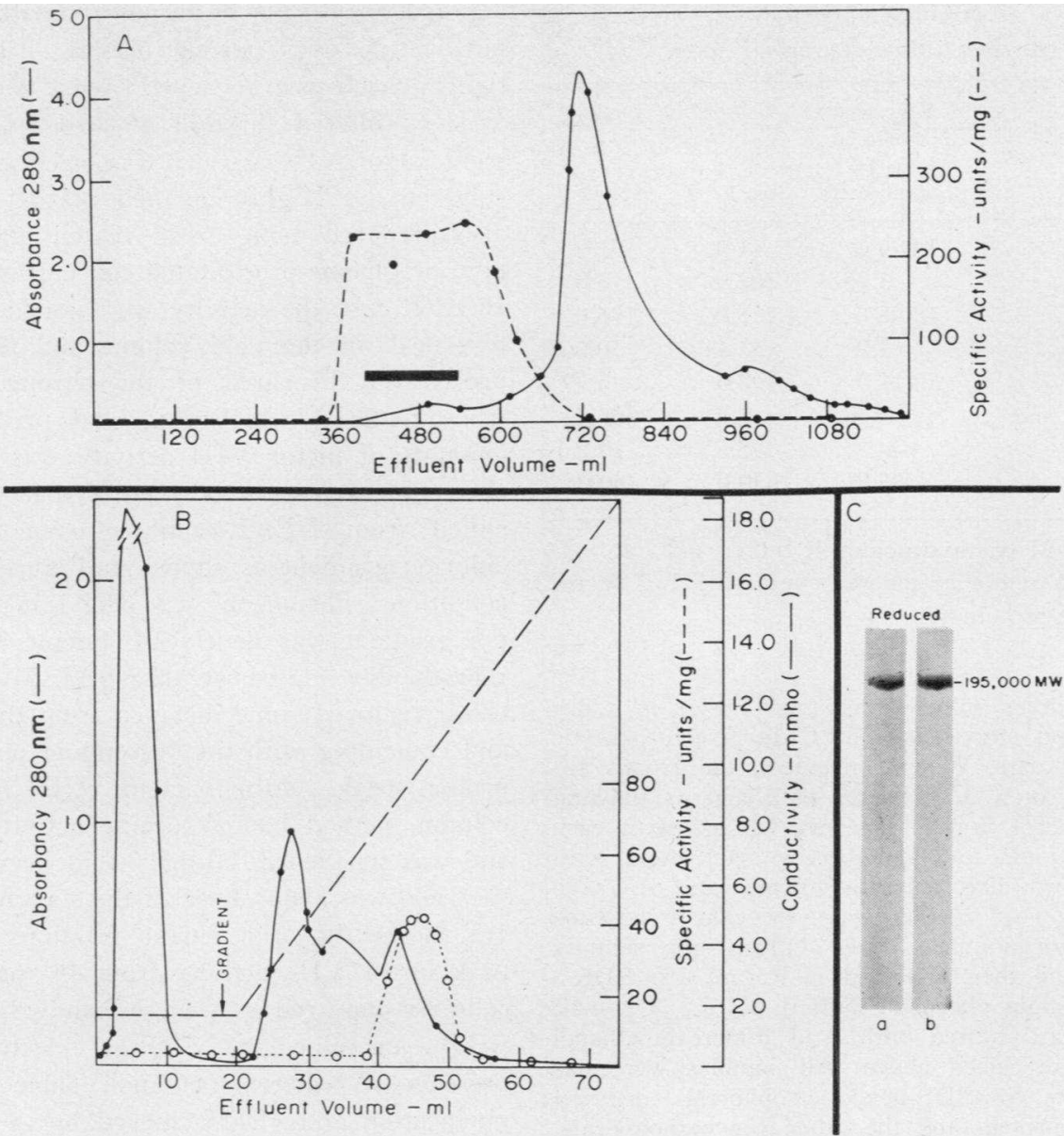

Figure 1 Panel A. 4\% agarose gel filtration of partially purified factor VIII (Step 1 factor VIII) (column size : $5 \times 92 \mathrm{~cm}$ ); the fractions beneath the horizontal bar were pooled and contained purified factor VIII (Step 2 factor VIII). Panel B. Gradient elution of partially purified factor VIII (Step 1) from DEAE-cellulose (column size $0.9 \times 10 \mathrm{~cm}$ ). The material coinciding with the descending limb of the last protein peak, and which maximally shortened the activated partial thromboplastin time, contained purified factor VIII (Step 2DEAE factor VIII). Panel C. SDS-gel electrophoresis of reduced factor VIII prepared from $4 \%$ agarose chromatography (gel $a$ ) or from DEAE-chromatography (gel $b$ ). MW, molecular weight.

the $4 \%$ agarose void volume protein in a collodion bag concentrator. By this method the initial $3-4 \mathrm{ml}$ of the void volume could be reduced to a volume of $100-500 \mu \mathrm{l}$, but with an estimated $50 \%$ loss of protein. Immunodiffusion studies of Step 1 and Step 2 normal and hemophilic factor VIII were performed in agarose, B grade, prepared as previously described (16). In each case, the wells were filled three times with $10 \mu l$ of a solution of Step 1 or Step 2 normal or hemophilic factor VIII containing about $1 \mu \mathrm{g} / \mathrm{ml}$.

Effect of thrombin on factor VIII. Using glycine-precipitated material, Step 1 normal factor VIII was prepared and further purified by elution from the $5 \times 92 \mathrm{~cm} 4 \%$ agarose column. Approximately $100 \mathrm{mg}$ of Step 1 material was dissolved in $12 \mathrm{ml}$ and applied to the column. The Step 2 factor VIII that was eluted in the void volume peak was pooled and then divided into $4 \mathrm{ml}$ aliquots in siliconized tubes. The final protein concentration of the pooled factor VIII was $5.7 \mu \mathrm{g} / \mathrm{ml}$; factor VIII activity was $0.52 \mathrm{U} / \mathrm{ml}$. The 4-ml aliquots were arranged in eight pairs. Purified human thrombin (also supplied by Dr. D. L. Aronson) was added to one tube of each pair to give a final concentration of $0.05 \mathrm{NIH} \mathrm{U} / \mathrm{ml}$, and an equal volume of buffer was added to the other tube of each pair. The paired mixtures were incubated at $37^{\circ} \mathrm{C}$ and at the end of each of the following time periods: $0.25,5,10,15,30,105,195$, and $284 \mathrm{~min}$, a pair of aliquots was assayed for factor VIII activity using three dilutions in the kaolin-activated, one-stage partial thromboplastin method. In this assay, control clotting times were as follows: $168 \mathrm{~s}$ for buffer alone, $156 \mathrm{~s}$ for a buffer solution containing $0.05 \mathrm{NIH}$ $\mathrm{U} / \mathrm{ml}$ of thrombin, and $129 \mathrm{~s}$ for a $1: 20$ dilution of the purified normal factor VIII solution. Immediately after a sample for assay had been removed from an incubation 
TABLE I

$4 \%$ Agarose Column Purification of Normal Factor VIII

\begin{tabular}{lccc}
\hline & $\begin{array}{c}\text { Glycine- } \\
\text { precipitated } \\
\text { factor VIII } \\
\text { concentrate* }\end{array}$ & Step 1 & Step 2 \\
\hline Volume, ml & $1,096.0$ & 14.0 & 92.0 \\
Activity, $\mathrm{C} / \mathrm{ml}$ & 3.93 & 302.0 & 13.0 \\
Total units & $4,308.0$ & $4,228.0$ & $1,196.0$ \\
Protein, mg/ml & 4.9 & 33.4 & 0.063 \\
Total protein, mg & $5,414.0$ & 468.0 & 5.79 \\
Specific activity, U/mg & 0.80 & 9.0 & 206.0 \\
Yield, Co & - & 98.5 & 29.2 \\
Purification-fold $\ddagger$ & 55.9 & 630.0 & $14,400.0$ \\
\hline
\end{tabular}

* Dissolved in $0.02 \mathrm{M}$ tris hydrochloride buffer, $\mathrm{pH}$ 7.4.

$\ddagger$ With respect to a reference plasma containing $1 \mathrm{U}$ factor $\mathrm{VIII} / \mathrm{ml}$ and $70 \mathrm{mg}$ protein $/ \mathrm{ml}$.

mixture, the remaining volume was quick-frozen in a dry ice-ethanol bath and stored at $-80^{\circ} \mathrm{C}$ for electrophoretic analysis at a later date. When convenient, the frozen aliquots were placed in a $37^{\circ} \mathrm{C}$ water bath and as thawing began, $0.5 \mathrm{ml}$ of $50 \%$ formic acid was mixed with each sample to lower the $\mathrm{pH}$ to 2 and thereby inactivate thrombin. ${ }^{2}$ Each sample was dialyzed in a volume ratio of $1: 200$ against three changes of distilled water, previously adjusted to $\mathrm{pH} 2.0$ with formic acid. After dialysis, the samples were lyophilized and then dissolved in $0.5 \mathrm{ml} 1 \% \mathrm{SDS}-5$ $\mathrm{M}$ urea-0.01 M sodium phosphate buffer, $\mathrm{pH}$ 7.1. One-half of this solution was reduced with $2 \mu 1 \quad \beta$-mercaptoethanol and incubated as described above. All samples were examined in duplicate by SDS-gel electrophoresis, one gel being stained for protein and the other for carbohydrate. Aliquots of Step 2 hemophilic factor VIII were examined similarly by incubating paired solutions containing 6.6 $\mu \mathrm{g} / \mathrm{ml}$ and $<1 \%$ activity with either a final thrombin concentration of $0.05 \mathrm{NIH} \mathrm{U} / \mathrm{ml}$ or with buffer only. At the completion of the incubation periods, each thrombinexposed sample and its control were assayed and then prepared for SDS-gel electrophoresis as described above.

The effect of thrombin concentration on factor VIII activity, after a constant incubation time, was also studied. In this experiment 4-ml aliquots of purified factor VIII containing $1.5 \mathrm{U} / \mathrm{ml}$ were incubated for $1 \mathrm{~min}$ at $37^{\circ} \mathrm{C}$ with the following final concentrations of purified human thrombin: $0.03,0.06,0.12,0.31$, and $0.62 \mathrm{U} / \mathrm{ml}$. At the end of the $1 \mathrm{~min}$ incubation period, each mixture was assayed by the two-stage method. It should be emphasized that the highest final concentration of thrombin used in the incubation mixtures did not affect the assay result when compared with a buffer control.

\section{RESULTS}

As previously reported (1-11), the partial purification of factor VIII by the method of Johnson, Newman, Howell, and Puszkin (2) followed by filtration through

${ }^{2}$ Lundblad, R. L., and L. C. Uhteg. 1972. Personal communication. Department of Biochemistry, University of North Carolina, Chapel Hill.
$4 \%$ agarose results in an approximate 7,000-10,000-fold purification over normal plasma. Figure $1 A$ shows a typical chromatogram when Step 1 normal human factor VIII is filtered through $4 \%$ agarose. In this experiment factor VIII activity was calculated using the twostage assay. Highly purified normal factor VIII was also prepared using fresh frozen plasma, cryoprecipitate, or glycine-precipitated concentrates of factor VIII. In each case the activity was associated with the protein peak in the roid rolume and the yield of active protein in this area of the chromatogram increased when material which contained progressively greater amounts of factor VIII activity was used. Besides gel chromatography, partially purified factor VIII was also eluted from DEAE-cellulose using a linear sodium chloride gradient as shown in Figure $1 B$. Under these conditions, fibrinogen was eluted in the first peak as the gradient was developed. Factor VIII activity was reproducibly eluted at $0.15-0.17 \mathrm{M}$ sodium chloride, being removed some distance from the fibrinogen peak and coinciding with the descending portion of the third protein peak. Normal factor VIII prepared by this column method had a specific activity of $30-50 \mathrm{U} / \mathrm{ml}$ and was purified 2,100-3,500-fold over starting plasma; the yield was about $3.6 \%$ of the starting activity. Figure $1 C$ shows that the eluant fractions which contained peak factor VIII activity from $4 \%$ agarose or DEAEcellulose appeared as a single band of 195,000 molecular weight examined by SDS-gel electrophoresis in the presence of $\beta$-mercaptoethanol. Table I summarizes the purification and yield achieved by the $4 \%$ agarose gel filtration method.

Fig. 2 shows the mean elution patterns when partially purified factor VIII from the six normal and six hemophilic volunteers was eluted from $4 \%$ agarose. The number of absorbancy units of Step 1 hemophilic factor VIII applied to the column was always slightly less than the amount of Step 1 normal factor VIII. Despite this, when partially purified hemophilic factor VIII was applied to the column, the amount of protein eluted in the void volume was not significantly different from that observed when partially purified normal factor VIII was chromatographed. All of the hemophiliacs had very similar quantities of void volume protein. However, striking differences were observed in the factor VIII activity in this region of the chromatograms. In these experiments the percent shortening of the partial thromboplastin time of hemophilic plasma given by an eluate fraction when compared with a buffer control was divided by the absorbance at $280 \mathrm{~nm}$ to generate an arbitrarily defined specific activity. As shown in Fig. 2, the amount of factor VIII activity associated with the void volume region in the elution pattern of the normal factor VIII was very significantly 


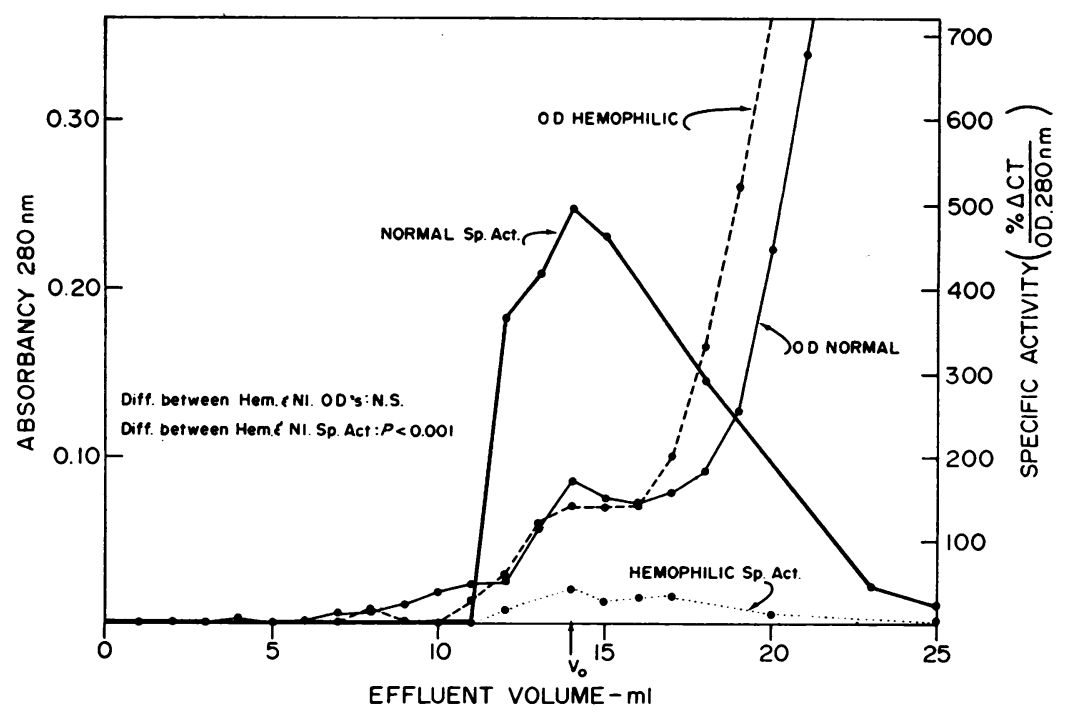

Figcre 2 Comparison of the mean elution patterns and the mean specific activities of individual samples of Step 2 factor VIII prepared separately from six normal plasmas and six hemophilic plasmas by filtration through $4 \%$ agarose (column size $0.9 \times 56 \mathrm{~cm}$ ). The mean amount of normal protein which appeared in the void volume was essentially identical to the mean amount of hemophilic protein. The specific activities were significantly different. It should be noted that each of the hemophilic plasmas contained virtually the same amount of void volume protein.

greater $(P<0.001)$ than in the case of the hemophilic factor VIII. The differences in the mean amount of protein or the mean amount of factor VIII activity between the six individual normal when compared with the six individual hemophilic preparations were examined using a tube-to-tube comparison of eluate fractions as well as a comparison of areas beneath the protein or activity peaks. In each case the same statistical results were obtained.

Subunit molecular weight of factor VIII. Fig. 3 shows typical sodium dodecyl sulfate polyacrylamide gel electrophoretic patterns of the protein peak associated with functional as well as nonfunctional factor VIII from gel filtration of normal and hemophilic concentrates. Gels $a$ and $b$, respectively, show that nonreduced normal or hemophilic factor VIII does not penetrate the top of $5 \%$ gels despite denaturation by $2 \%$ SDS-5 $\mathrm{M}$ urea. Even after it was dissolved in $6 \mathrm{M}$ guanidine hydrochloride and dialyzed into $2 \%$ SDS, neither protein entered $5 \%$ polyacrylamide gels. However, gels $c$ and $d$ show that after reduction of either normal or hemophilic factor VIII by $0.1 \mathrm{M} \beta$-mercaptoethanol, a major protein band of 195,000 molecular weight was observed in each case. Gels $e$ and $f$ show that this band, whether from normal or hemophilic protein, contained carbohydrate when stained with the periodic acid-Schiff reagent. It should be noted that despite reduction by concentrations of $\beta$-mercaptoethanol of $0.4 \mathrm{M}$ for as long as $40 \mathrm{~h}$ in $2 \%$ SDS or $6 \mathrm{M}$ guanidine hydrochloride, no further decrease occurred in the molecular weight of this subunit species.

Figure $4 A$ shows the standard curve for molecular weight determination by the SDS-gel electrophoretic method. By this technique both reduced normal and hemophilic factor VIII had molecular weights of 195 ,000 . Figure $4 B$ depicts the logarithmic relationship be-

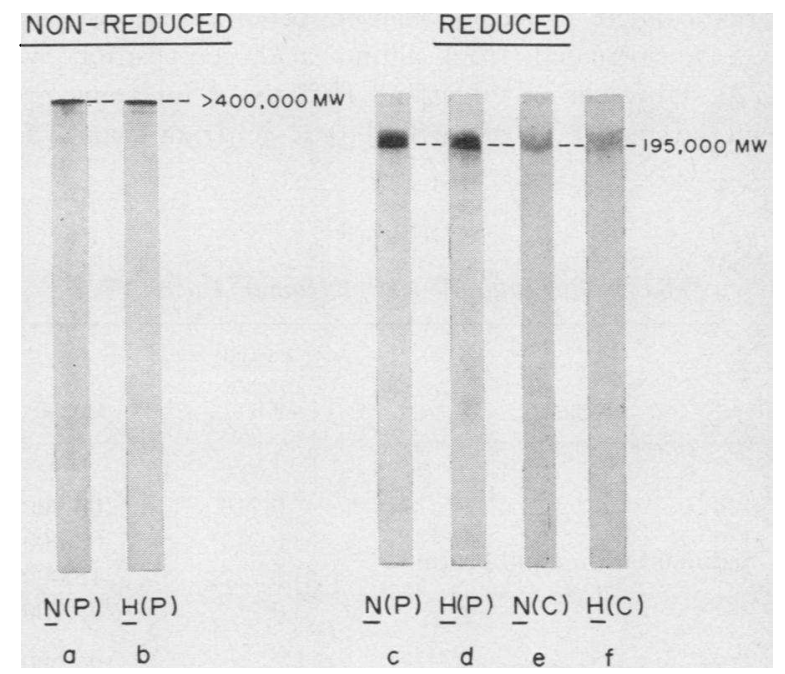

FIGURE 3 SDS-gel electrophoresis of non-reduced and reduced normal, $N$, and hemophilic, $H$, factor VIII. Gels indicated by $(P)$ were stained for protein; gels indicated by (C) were stained for carbohydrate. MW, molecular weight. 

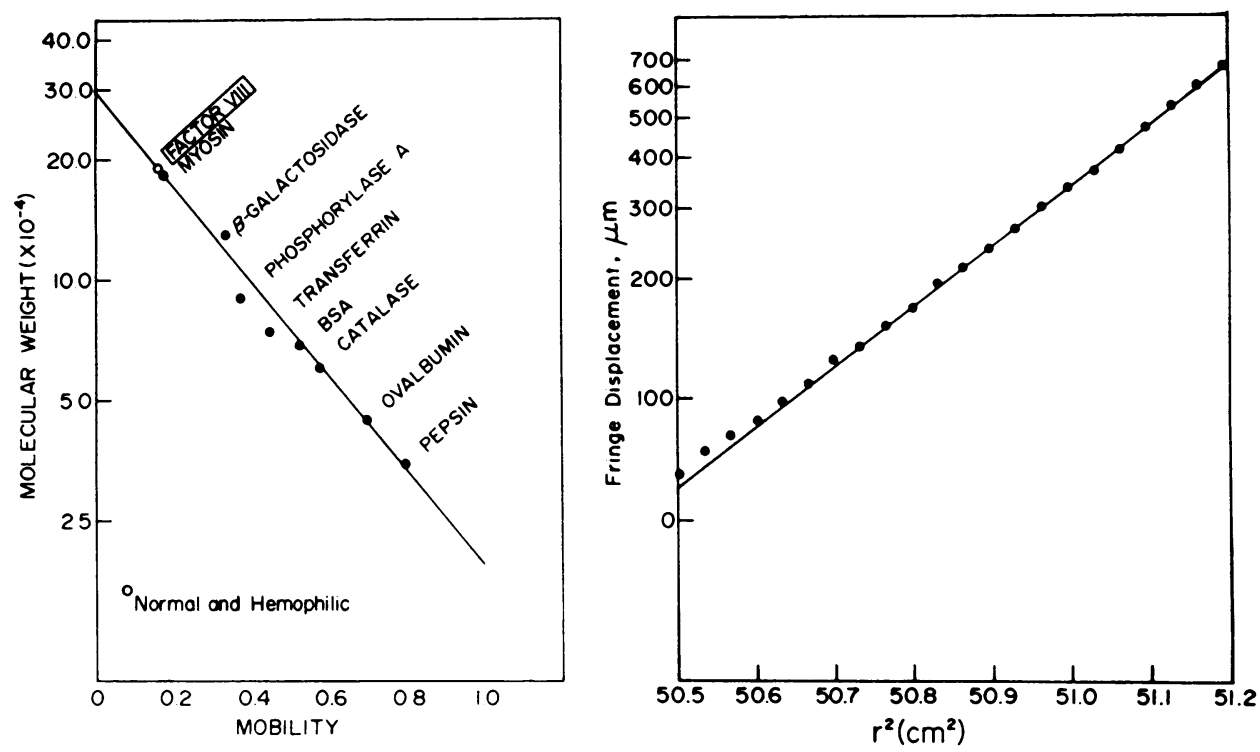

Figlre 4 Pancl A. SDS-gel electrophoretic standard curve for determination of the subunit molecular weight of reduced normal and hemophilic factor VIII. The mobilities of the normal and hemophilic factor VIII subunits were identical. Pancl B. Sedimentation equilibrium of reduced normal factor VIII in 5.6 M guanidine hydrochloride. See text for details.

tween the fringe displacement and the square of the distance from the center of rotation when the factor VIII subunit is analyzed by sedimentation equilibrium ultracentrifugation. Although the linear relationship from cell bottom to less than $100 \mu m$ fringe displacement found by this technique indicates homogeneity of the factor VIII subunit, calculation of an exact molecular weight depends upon knowledge of the partial specific volume $(\vec{v})$ for the subunit. Therefore Table II gives a list of molecular weights for different $\bar{v}$ values. The $\overline{\mathbf{v}}$ calculated from amino acid composition was 0.722 . However in guanidine $\mathrm{HCl}$ the $\bar{v}$ for some proteins is smaller by about $0.01-0.02 \mathrm{cc} /$ gram than $\bar{v}$ for

TABLE II

Subunit Molecular Weight of Human Factor VIII

\begin{tabular}{|c|c|c|}
\hline Method & $\begin{array}{c}\text { Partial } \\
\text { specific } \\
\text { volume, } \bar{Y}\end{array}$ & Mol wt \\
\hline \multirow{5}{*}{ Sedimentation equilibrium } & $c c_{;}^{\prime} g$ & \\
\hline & 0.710 & 200,000 \\
\hline & $0.712^{*}$ & 202,000 \\
\hline & $0.722 \ddagger$ & 215,000 \\
\hline & 0.730 & 226,000 \\
\hline SDS-gel electrophoresis & - & 195,000 \\
\hline
\end{tabular}

* Corrected $\bar{v}$ for preferential binding of guanidine hydrochloride (34).

$\ddagger$ Calculated from the amino acid composition (33). the same protein in dilute aqueous salt solution (35). When arbitrarily corrected for this effect, the calculated molecular weight from sedimentation equilibrium data becomes $\sim 202,000$ for the normal factor VIII subunit. This value is in good agreement with the 195,000 molecular weight obtained for both the normal and hemophilic factor VIII subunit by SDS-gel electrophoresis.

Isoelectric focusing. Fig. 5 shows superimposed scans of reduced and alkylated normal and hemophilic factor VIII when analyzed by isoelectric focusing in polyacrylamide gels. Although slightly diffuse, both the normal and hemophilic factor VIII subunits had identical mobilities when examined by this method.

Amino acid composition. Table III shows the amino acid analysis of normal human factor VIII. The values listed in the first column are the averages of each residue when calculated from the values obtained by analyses of duplicate samples at three different times of hydrolysis. Results are expressed in terms of residues of each amino acid per subunit molecular weight. The second column shows the amino acid composition of normal factor VIII reported by Hershgold, Davison, and Janszen (8), but recalculated on the basis of 195,000 subunit molecular weight so that our data and theirs may be compared. With the exception of half-cystine, our data and theirs are similar with about half the residues being within $\pm 5 \%$. It should be noted that in the present study, half-cystine was determined after performic acid oxidation which tends to prevent the under- 
estimation of this residue. Since values for valine, isoleucine, and leucine increase with time, these were determined after hydrolysis for $120 \mathrm{~h}$.

Immunological studies. The results of these studies essentially confirmed those previously reported by Zimmerman et al (16). Human plasma which contained a high titer of a spontaneously formed inhibitor to factor VIII prolonged the partial thromboplastin time of the most purified fractions of normal factor VIII from 60 to $165 \mathrm{~s}$; a buffer control was $155 \mathrm{~s}$. Bands of identity were observed when absorbed rabbit antiserum to purified normal factor VIII was diffused against Step 1 normal factor VIII, as well as Step 1 hemophilic factor VIII. Functionally, when a $1: 2$ dilution of unabsorbed or absorbed antiserum to Step 2 normal factor VIII was incubated with normal human plasma at $37^{\circ} \mathrm{C}$ for $2 \mathrm{~h}$, the effectiveness of the normal plasma in correcting the partial thromboplastin time of hemophilic plasma was markedly diminished as evidenced by a prolongation of the PTT from $79.2 \mathrm{~s}$ to 104.2 and $125.9 \mathrm{~s}$, respectively.

Effect of thrombin on factor VIII. Fig. 6 shows the response of purified normal factor VIII to trace quantities of thrombin with time. Percent factor VIII activity was estimated by the one-stage partial thromboplastin time method. The value shown on the ordinate is the percent activity given by the purified factor VIIIthrombin mixture normalized to the activity given by purified factor VIII alone. Buffer, which contained the same final thrombin concentrations as did the factor VIII-thrombin mixture, gave clotting times that were not significantly different from those of buffer alone. As can be seen, final thrombin concentrations of 0.05 $\mathrm{U} / \mathrm{ml}$ rapidly activated and then progressively inactivated factor VIII. Purified hemophilic factor VIII, however, activated only slightly in response to thrombin. The reduced SDS-gels in Fig. 6 show that neither activation or inactivation of factor VIII was accompanied by a change in subunit molecular weight. In this preparation two very faint bands of molecular weight approximately 100,000 and 130,000 were inconsistently observed; their intensity was not affected by exposure to thrombin. Experiments in progress indicate that these two bands, which can occasionally be found in trace amount in purified factor VIII, result from degradation of factor VIII by plasmin. In addition the intensity of the carbohydrate stain did not vary during activation and inactivation. Similarly, changes in carbohydrate staining intensity, as assessed by the periodic acid-Schiff reagent, were not detectable during incubation of hemophilic factor VIII with thrombin. Fig. 7 demonstrates the effect of different thrombin concentrations when incubated with a constant concentration of factor VIII for $1 \mathrm{~min}$ at $37^{\circ} \mathrm{C}$. In this ex-

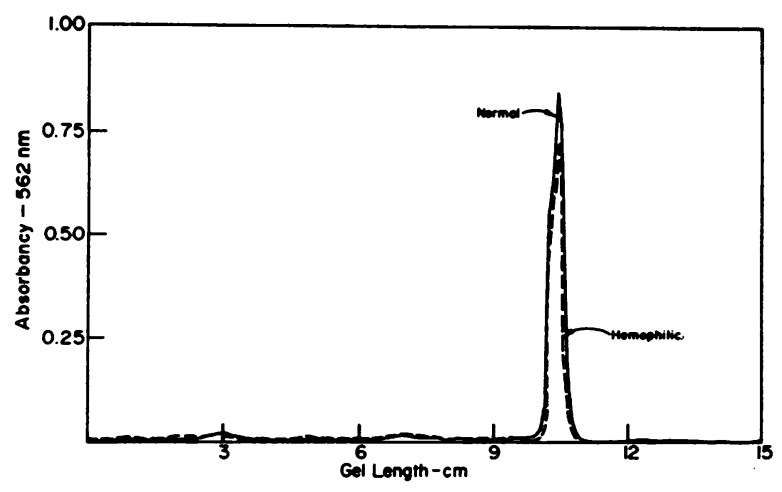

Figtre 5 Gel scan of the reduced and carboxymethylated subunit from normal and hemophilic factor VIII after isoelectric focusing in polyacrylamide gels. The top of the gel is represented by the 0 point on the abscissa.

periment, factor VIII activity was estimated with the two-stage assay, especially modified so as to be sensitive to thrombin-induced activation. The first stage incubation time was held at $2 \mathrm{~min}$ in order to optimally demonstrate the thrombin effect on factor VIII. Were this time to have been prolonged the inactivation of factor VIII by thrombin as well as by plasmin (present in the serum components of this assay) (41) might have obscured the immediate activation of factor VIII by thrombin. The concentrations of thrombin used in this study did not affect the results of the two-stage assay when tested in buffer alone. Clearly at a final thrombin

TABLE III

Amino Acid Composition of Human Factor VIII*

\begin{tabular}{lcr}
\hline \multicolumn{1}{c}{ Amino acid } & $\begin{array}{c}\text { Present } \\
\text { study }\end{array}$ & $\begin{array}{c}\text { Hershgold } \\
\text { et al. (8) }\end{array}$ \\
\hline Lysine & 95.94 & 99.06 \\
Histidine & 53.04 & 33.74 \\
Arginine & 94.38 & 85.02 \\
Aspartic acid & 162.63 & 154.25 \\
Threonine & 99.65 & 99.06 \\
Serine & 129.87 & 122.85 \\
Glutamic acid & 224.06 & 201.24 \\
Proline & 117.00 & 101.60 \\
Glycine & 131.63 & 128.70 \\
Alanine & 96.92 & 105.89 \\
Half-cystine & $120.71 \ddagger$ & 45.83 \\
Valine & $151.71 \S$ & 116.22 \\
Methionine & 24.77 & 19.31 \\
Isoleucine & $62.21 \S$ & 61.82 \\
Leucine & 134.36 & 133.38 \\
Tyrosine & 34.71 & 44.85 \\
Phenylalanine & 49.34 & 51.68 \\
\hline
\end{tabular}

* Residues per 195,000 molecular weight subunit.

$\ddagger$ Determined after oxidation.

$\$ 120$ h hydrolysis. 


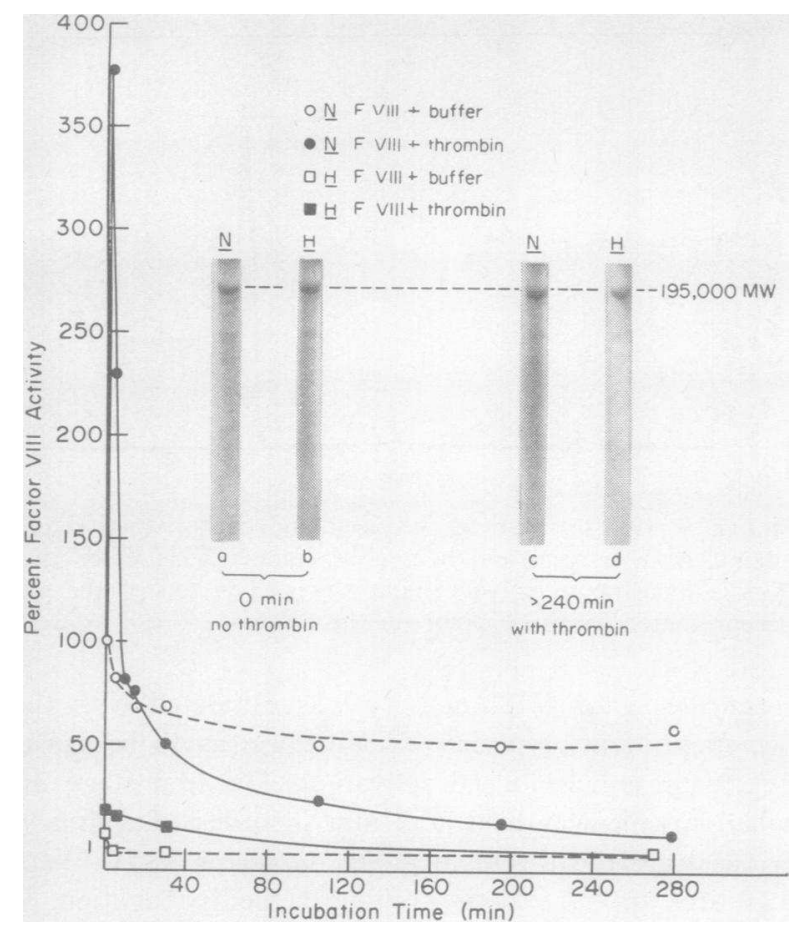

FIGLRE 6 The effect of thrombin on the activity of purified normal and hemophilic factor VIII. Activity was assessed by the one-stage activated partial thromboplastin time assay. Gel $a$ and gel $b$ compare the reduced SDS-gel electrophoretic patterns of normal and hemophilic factor VIII before the addition of thrombin at 0 time. Gel $b$ and gel $c$ compare the mobilities of reduced normal $(N)$ and hemophilic $(\mathrm{H})$ factor VIII after each had been exposed to $0.05 \mathrm{U} / \mathrm{ml}$ of thrombin $>240 \mathrm{~min}$. No detectable change in the subunit molecular weight (MW) of normal factor VIII occurred despite activation and inactivation during this time period. Similarly no apparent change in the molecular weight of the hemophilic factor VIII subunit occurred during exposure to thrombin.

concentration of about $0.03 \mathrm{U} / \mathrm{ml}$, activation or factor VIII occurred; however, at thrombin concentrations above $0.312 \mathrm{U} / \mathrm{ml}$, factor VIII activity was rapidly destroyed.

\section{DISCUSSION}

The studies reported here expand previous observations (1-11) concerning certain properties of normal, functional human factor VIII, and in addition, support immunological data (13-18) indicating that nonfunctional factor VIII is present in classical hemophilia. Several investigators have shown that highly purified factor VIII can be prepared by filtering factor VIII concentrates through columns of $2-4 \%$ agarose as the final purification step (1-11). As confirmed in the present study, this latter purification step results in an approximate 10,000 -fold increase in specific activity with re- spect to normal plasna. When glycine-precipitated factor VIII was the starting material, the yield of normal factor VIII after the $4 \%$ agarose chromatography step was about $29 \%$ as shown in Table I. When freshfrozen plasma was used, the yield of factor VIII after agarose chromatography was somewhat lower, being about $10 \%$ of the activity in the starting plasma; this value is in good agreement with previously reported recoveries $(8,10,20)$. Moreover, the recovery of purified factor VIII from agarose columns was usually about this latter value despite whether the starting material was glycine-precipitated factor VIII, cryoprecipitate, or fresh-frozen plasma. After rechromatography on agarose gel columns, a single, symmetrical peak was observed in the void volume region and no further purification was achieved.

In the case of DEAE-cellulose chromatography, however, the yield of purified factor VIII was much lower -approximately $3.6 \%$ of the activity in the starting plasma. Conceivably factor VIII prepared by this method is partially degraded or modified with a resulting loss of activity during the ion exchange chromatography step. The important feature of the DEAEcellulose step, however, was the demonstration that factor VIII activity coincided with the elution of a protein which, after reduction, was found to contain a single subunit species identical in molecular weight to the subunit species of factor VIII prepared by agarose gel filtration; in addition both contained carbohydrate.

Since the yield of normal factor VIII appeared to be consistently proportional to the activity in the starting material and since Zimmerman et al. (16) observed that hemophilic plasma contains normal amounts of nonfunctional factor VIII-like antigen, the yield of void volume protein and factor VIII activity was examined and compared for six normal men and six classical hemophiliacs. It should be noted that since yields of factor VIII are calculated on the basis of activity, no such calculations could be made for hemophilic plasmas. It was assumed that if a normal level of nonfunctional, abnormal factor VIII protein was present in hemophilic plasmas, fractionation procedures identical to those used for normal plasmas might result in the recovery of final quantities of hemophilic factor VIII similar to those of normal factor VIII. This indeed proved to be the case. As shown in Fig. 2, when material prepared from hemophilic plasma was chromatographed on $4 \%$ agarose, a protein peak was observed in the void volume for each hemophilic preparation just as in the case of each normal preparation. While the expected amount of protein was observed, however, very little factor VIII activity was found in this peak for the hemophilic samples. The fact that some activity was noted despite $<1-5 \%$ activity levels in the six hemo- 
philic plasmas is not surprising in view of the concentration effect achieved by the purification method.

From the observation that factor VIII activity occurs in or very near the void volume during agarose chromatography, the native molecular weight of human factor VIII has been estimated at approximately 1-2 million $(3,8,10)$. Hershgold et al. (8) reported an estimated $s_{20, w}$ of 16.3 in $6 \mathrm{M}$ guanidine hydrochloride which tends to substantiate its large size and suggests that the native molecule is held together by covalent bonds. The polyacrylamide gel electrophoretic results of Kass, Ratnoff, and Leon (3) and Hershgold et al. (8) suggested that the factor VIII molecule was too large to enter even a 3\% polyacrylamide gel. Our initial observations suggested this to be the case even after exposing factor VIII to denaturing solvents such as guanidine hydrochloride and sodium dodecyl sulfate (5). Since then Marchesi, Shulman, and Gralnick have reported similar findings (11), and more recently Schmer, Kirby, Teller, and Davie (10) have observed that bovine factor VIII does not enter a $3.25 \%$ polyacrylamide gel even in the presence of $8 \mathrm{M}$ urea. These studies all indicated that any subunits in the factor VIII molecule must be joined by covalent bonds. When factor VIII was reduced by $\beta$-mercaptoethanol and examined by SDS-gel electrophoresis a single band was observed with a molecular weight initially estimated to be about 215,000 daltons. Since then the subunit molecular weight of rabbit myosin, an important standard in our SDS-gel electrophoretic analyses, has been more accurately determined (28) and we now report the molecular weight of the factor VIII subunit to be 195,000 (41). It is to be emphasized that on SDS-gels, the factor VIII subunit had a mobility which was consistently less than the myosin subunit of 194,000 molecular weight. As shown in Fig. 3, normal and hemophilic purified factor VIII had identical subunit molecular weights and moreover, both contained carbohydrate when stained by the periodic acid-Schiff reagent. To date, there is little consensus about the actual size of the human factor VIII subunit. While our finding of 195,000 molecular weight for the factor VIII subunit is smaller than the value of 240,000 molecular weight reported by Marchesi et al. (11), it is of interest that both values suggest that the human factor VIII molecule is composed of several very large subunit polypeptide chains. The differences between the presently reported molecular weight of 195,000 and the value of 240,000 obtained by Marchesi et al (11) can be explained by their use of non-reduced fibrinogen as a marker for the high molecular weight portion of the standard curve. Of concern are the completely divergent results of Hershgold et al. (8) which suggest that the human factor VIII molecule is composed of 22,000

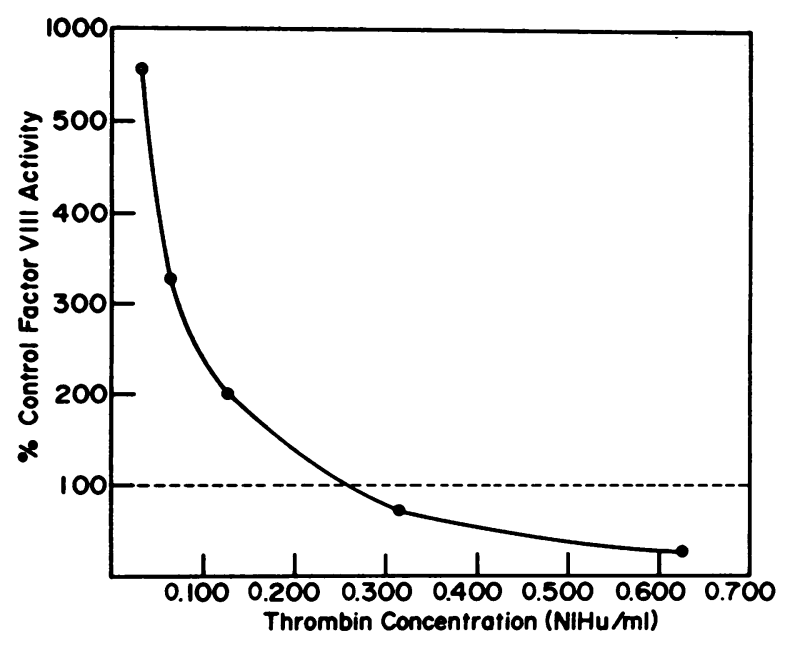

Figure 7 The immediate effect of thrombin concentration on the activation and inactivation of purified normal factor VIII. Factor VIII activity is expressed as a percentage of the purified factor VIII activity prior to the addition of thrombin.

molecular weight subunits held together by disulfide bonds. This latter value cannot be evaluated fully since details of the experiments have not yet been published. From their preliminary molecular weight determinations by sedimentation equilibrium analysis, Schmer et al. (10) have recently suggested that the subunits of bovine factor VIII may not be identical and instead, may range in size from 85,000 to 198,000 . However, when their bovine factor VIII preparation was reduced and analyzed in an $8 \mathrm{M}$ urea-3.25\% polyacrylamide gel electrophoretic system, a single band of relatively low mobility was observed, thus suggesting that the bovine factor VIII subunit is indeed large and probably homogeneous in size. Moreover, the results of sedimentation equilibrium ultracentrifuge analyses in the present study indicate that the human factor VIII subunit is homogeneous by size, and has an approximate molecular weight of 202,000 which is very close to the value obtained by SDS-gel electrophoresis.

In electrophoretic systems in which mobility is primarily a function of charge rather than size, the patterns of purified factor VIII preparations have been difficult to interpret since a substantial amount of sample usually remained at the origin $(3,8)$. This is probably due to the large size of the molecule which prevents its penetrating the usual $5 \%$ polyacrylamide gels $(5,10$, 11). In the present study non-reduced factor VIII did not penetrate even the most loosely cross-linked acrylamide gels $(3.36 \%)$. When reduced and alkylated and the examined by isoelectric focusing, however, a single band, albeit slightly diffuse, was observed. A band of identical mobility was noted when reduced and alky- 
lated purified hemophilic factor VIII was examined by isoelectric focusing. These latter observations are in accord with those reported by Schmer et al. (10) who found that reduced and alkylated bovine factor VIII migrated as a single spot in zone electrophoresis. When the results of SDS-gel electrophoresis, zone electrophoresis and isoelectric focusing are combined, it appears that the repetitive subunits in human or bovine factor VIII are identical in size and charge. This raises doubt concerning sedimentation equilibrium data which suggest the molecular weights of bovine factor VIII subunits vary from 85,000 to 198,000 (10) since molecular weight differences of this magnitude should be accompanied by easily detectable charge differences on zone electrophoresis or isoelectric focusing.

The amino acid composition data suggest that the aromatic residue content of normal human factor VIII is lower than usually observed for most proteins; Schmer et al. have reported similar findings for bovine factor VIII (10). These data provide a likely explanation for the past observations of poor absorption at 280 $\mathrm{nm}$ for factor VIII preparations. Table III compares the amino acid composition of the human factor VIII subunit from the present study with that calculated from the data of Hershgold et al (8) on the basis of a subunit molecular weight of 195,000 . As can be seen, the compositions are fairly similar except for marked discrepancies in the histidine values and half-cystine content. The latter variation can be explained since in the present study half cystine was determined after performic acid oxidation and therefore can be considered the more reliable value. We have no explanation for the discrepant histidine values. The high degree of similarity between the amino acid compositions in the present study and those reported by Hershgold et al. (8) suggest that the same protein was being examined. Because of the obvious difficulty in obtaining adequate quantities of hemophilic plasma, purified hemophilic factor VIII could not be prepared in amounts sufficient for determination of amino acid composition.

Our immunological studies largely confirm those reported by others (13-18). A spontaneously formed human factor VIII inhibitor as well as antiserum prepared in rabbits to highly purified human factor VIII clearly neutralized factor VIII activity in specific factor VIII assays. Moreover, identical precipitin bands were noted when the antiserum was tested against purified normal or hemophilic factor VIII by immunodiffusion.

While other workers have reported that factor VIII can be activated and subsequently inactivated by trace amounts of thrombin (41-46), the present study represents the first reported attempt to determine if changes in factor VIII structure occur concomitantly with changes in function. In this study, no detectable change in subunit molecular weight or change in intensity of protein or carbohydrate staining was discernible with activation and inactivation of normal factor VIII by thrombin. Similar treatment of purified factor VIII from hemophilic plasmas demonstrated only a slight increase in activity with respect to protein concentration and no detectable structural changes. Because of the well-documented proteolytic activity of thrombin toward fibrinogen (47) and fibrin stabilizing factor (48), it seems likely that catalytic amounts of thrombin affect factor VIII activity by similar specific proteolytic modification of the factor VIII subunits. This hypothesis receives additional support from the observation that trypsin, a known protease of lesser specificity, is also capable of activating and inactivating factor VIII without initial structural change in the factor VIII subunit: extensive degradation, however, occurs following prolonged incubation (41). These observations suggest that both activation and inactivation of normal factor VIII may occur by cleavage of peptide bonds resulting in fragments, the sum of which molecular weights is less than about 10,000 , since the limits of resolution of the SDS-gel system are about $\pm 5 \%$. It seems probable then, that thrombin produces an initial increase in factor VIII activity by cleaving a very small peptide from the factor VIII molecule. The subsequent complete loss of factor VIII activity may then result from additional cleavage of small peptide(s) by thrombin.

In conclusion the results of the present study indicate the following: (a) human factor VIII is a plasma glycoprotein with a molecular weight of 1-2 million; it is composed of 195,000 molecular weight repetitive subunits, most likely totaling 5-10, joined by disulfide bonds; $(b)$ hemophilic factor VIII appears identical to normal factor VIII in subunit molecular weight, carbohydrate content, electrical charge, and antigenic determinants; (c) the activation and inactivation of normal factor VIII by thrombin without an apparent change in molecular weight and without the appearance of additional peptide bands suggest that the release of peptides totaling less than 10,000 molecular weight per factor VIII subunit may account for both events.

\section{ACKNOWLEDGMENTS}

This work was supported by a Clinical Investigatorship (Dr. McKee), Veterans Administration and a research grant from the National Heart and Lung Institute, National Institutes of Health (HE-05736).

The authors wish to thank Mrs. Augusta S. Dingeldein and Dr. Ross Tye for valuable advice and assistance with portions of these studies.

\section{REFERENCES}

1. Hershgold, E. G., L. Silverman, A. Davison, and M. Jansen. 1967. Native and purified factor VIII: molecular 
and electron microscopical properties and a comparison with hemophilic plasma. Fcd. Proc. 26: 488.

2. Johnson, A. J., J. Newman, M. B. Howell, and S. Puszkin. 1967. Purification of antihemophilic factor (AHF) for clinical and experimental use. Thromb. Diath. Haemorrh. 26 (Suppl.) : 377.

3. Kass, L., O. D. Ratnoff, and M. A. Leon. 1969. Studies on the purification of antihemophilic factor (factor VIII). I. Precipitation of antihemophilic factor by concanavalin A. J. Clin. Invest. 48: 351.

4. Ratnoff, O. D., L. Kass, and P. D. Lang. 1969. Studies on the purification of antihemophilic factor (factor VIII). II. Separation of partially purified antihemophilic factor by gel filtration of plasma. J. Clin. Invest. 48: 957.

5. Shapiro, G. A., and P. A. McKee. 1970. Demonstration of a nonfunctional antihemophilic factor (factor VIII) in classic hemophilia. Clin. Res. 18: 615.

6. Van Mourik, J. A., and I. A. Mochtar. 1970. Purification of human antihemophilic factor (factor VIII) by gel chromatography. Biochem. Biophys. Acta. 221: 677.

7. Green, D. 1971. A simple method for the purification of factor VIII (antihemophilic factor) employing snake venom. J. Lab. Clin. Med. $77: 153$.

8. Hershgold, E. J., A. M. Davison, and M. E. Janszen. 1971. Isolation and some chemical properties of human factor VIII (antihemophilic factor). J. Lab. Clin. Med. $77: 185$.

9. Van Mourik, J. A., B. N. Bouma, J. J. Sixma, and I. A. Mochtar. Purification and characterization of antihemophilic factor $A$ and von Willebrand factor. The International Society on Thrombosis and Haemostasis. 2nd Congress, 12-16 July 1971. Villco trykkeri, Oslo, Norway. 38 .

10. Schmer, G., E. P. Kirby, D. C. Teller, and E. IV. Davie. 1972. The isolation and characterization of bovine factor VIII (antihemophilic factor). J. Biol. Chem. $247: 2512$.

11. Marchesi, S. L., N. R. Shulman, and H. R. Gralnick. 1972. Studies on the purification and characterization of human factor VIII. J. Clin. Invest. 51: 2151.

12. Hershgold, E. J. 1971. The subunit structure of human factor VIII (antihemophilic factor). Fed. Proc. 30: 540.

13. Shanberge, J. N., and I. Gore. 1957. Studies on the immunologic and physiologic activities of antihemophilic factor (AHF). J. Lab. Clin. Med. 50: 954.

14. Hoyer, L. W., and R. T. Breckenridge. 1968. Immunologic studies on antihemophilic factor (AHF, factor VIII) : cross-reacting material in a genetic variant of hemophilia A. Blood. 32: 962.

15. Bennett, E., and E. R. Huehns. 1970. Immunological differentiation of three types of haemophilia and identification of some female carriers. Lancet. 2: 956.

16. Zimmerman, T. S., O. D. Ratnoff, and A. E. Powell. 1971. Immunologic differentiation of classic hemophilia factor VIII deficiency) and Von Willebrand's diseasewith observations on combined deficiencies of antihemophilic factor and proaccelerin (factor V) and on an acquired circulating anticoagulant against antihemophilic factor. J. Clin. Invest. $50: 244$.

17. Stites, D. P., E. J. Hershgold, J. D. Perlman, and H. H. Fudenberg. 1971. Factor VIII detection by hemagglutination inhibition: Hemophilia $\mathrm{A}$ and Von Willeband's disease. Science (Wash. D. C.). 171: 196.

18. Meyer, D., J. M. Lavergne, M. J. Larrieu, and F. Josso. 1972. Cross-reacting material in congenital factor VIII deficiencies (haemophilia A and Von Willebrand's disease). Thromb Res. 1: 183.

19. Langdell, R. D., R. H. Wagner, and K. M. Brinkhous. 1953. Effect of antihemophilic factor on one-stage clotting tests. J. Lab. Clin. Med. 41: 637.

20. Newman, J., A. J. Johnson, M. H. Karpatkin, and S. Puszkin. 1971. Methods for the production of clinically effective intermediate- and high-purity factor-VIII concentrates. Br.J. Haematol. $21: 1$.

21. Ratnoff, O. D., R. E. Botti, R. T. Breckenridge, and A. S. Littell. 1964. Some problems in the measurement of antihemophilic activity. In The Hemophilias. K. M. Brinkhous, editor. The University of North Carolina Press, Chapel Hill. 3.

22. Waddell, W. J. 1956. A simple ultraviolet spectrophotometric method for the determination of protein. J. Lab. Clin. Med. $48: 311$.

23. Lowry, O. H., N. J. Rosebrough, A. L. Farr, and R. J. Randall. 1951. Protein measurement with folin phenol reagent. J. Biol. Chem. 193: 265.

24. Weber, K., and M. Osborn. 1969. The reliability of molecular weight determinations by dodecyl sulfatepolyacrylamide gel electrophoresis. J. Biol. Chem. 244: 4406.

25. McKee, P. A., P. Mattock, and R. L. Hill. 1970. Subunit structure of human fibrinogen, soluble fibrin and cross-linked insoluble fibrin. Proc. Natl. Acad. Sci. U.S. A. $66: 738$.

26. Schwartz, M. L., S. V. Pizzo, R. L. Hill, and P. A. McKee. 1971. The effect of fibrin-stabilizing factor on the subunit structure of human fibrin. J. Clin. Invest. 50: 1506 .

27. Paterson, B., and R. C. Strohman. 1970. Myosin structure as revealed by simultaneous electrophoresis of heavy and light subunits. Biochemistry. 9: 4094.

28. Gazith, J., S. Himmelfarb, and W. F. Harrington. 1970. Studies on the subunit structure of myosin. J. Biol. Chem. $245: 15$.

29. Trayer, H. R., Y. Nozaki, J. A. Reynolds, and C. Tanford. 1971. Polypeptide chains from human red blood cell membranes. J. Biol. Chem. 246: 4485.

30. Fish, W. W., K. G. Mann, and C. Tanford. 1969. The estimation of polypeptide chain molecular weights by gel filtration in $6 \mathrm{M}$ guanidine hydrochloride. J. Biol. Chem. 244: 4989.

31. Zacharius, R. M., T. E. Zell, J. H. Morrison, and J. J. Woodlock. 1969. Glycoprotein staining following electrophoresis on acrylamide gels. Anal. Biochem. 30: 148.

32. Hirs, C. H. W. 1967. Determination of cystine as cysteic acid. Methods Enzymol. 11: 59.

33. Yphantis, D. A. 1964. Equilibrium ultracentrifugation of dilute solutions. Biochemistry. 3: 297.

34. Cohn, E. J., and J. T. Edsall. 1965. Density and apparent specific volume of proteins. In Proteins, Amino Acids and Peptides. E. J. Cohn and J. T. Edsall, editors. Hafner Publishing Co., Inc., New York. 370.

35. Hade, E. P. K., and C. Tanford. 1967. Isopiestic compositions as a measure of preferential interactions of macromolecules in two-component solvents. Application to proteins in concentrated aqueous cesium chloride in guanidine hydrochloride. J. Am. Chem. Soc. 89: 5034.

36. Marler, E., and C. Tanford. 1964. The molecular weight of the polypeptide chains of L-glutamate dehydrogenase. J. Biol. Chem. 239: 4217.

37. Marler, E., C. A. Nelson, and C. Tanford. 1964. The 
polypeptide chains of rabbit $\gamma$-globulin and its papaincleaved fragments. Biochemistry. 3: 279.

38. Hirs, C. H. W. 1967. Reduction and S-carboxymethylation of proteins. Mcthods Ensy'mol. 11: 199.

39. Hayes, M. B., and D. Wellner. 1969. Microheterogeneity of L-amino acid oxidase. J. Biol. Chem. 244: 6636.

40. Roberts, H. R., M. B. Scales, J. T. Madison, W. P. Webster, and G. D. Penick. 1965. A clinical and experimental study of acquired inhibitors to factor VIII. Blood. 26: 805.

41. Andersen, J. C., and P. A. McKee. 1972. The effects of proteolytic enzymes on the coagulant properties and molecular structure of human factor VIII. Circ. Suppl. 2. $46: 52$.

42. Biggs, R., and R. G. McFarlane. 1965. The reaction between factors VIII and IX to form a factor $\mathrm{X}$ activator. Thromb. Diath. Hacmorrh. 17 (Suppl.): 23.

43. Ozge-Anwar, A. H. 1970. Slow activation of factor VIII by thrombin and other enzymes at low temperature. Scand. J. Haematol. $7: 5$.
44. Osterud, B., S. I. Rapaport, S. Schiffman, and M. M. Y. Chong. 1971. Formation of intrinsic factor-X-activator activity, with special reference to the role of thrombin. Br. J. Hacmatol. $21: 643$.

45. Legaz, M., G. Schmer, and E. Davie. 1972. Characterization of human factor VIII (antihemophilic factor). Circ. Suppl. 2. 46: 53.

46. Thompson, A. R., M. Legaz, and R. B. Counts. 1972. Proteolytic modification of factor VIII. Abstracts of the 15th Annual Meeting of the American Society of Hematology. No. 273. Faculty Press, Brooklyn, N. Y. 124.

47. Gladner, J. A. 1968. The action of thrombin on fibrinogen. In Fibrinogen. Koloman Laki, editor. Marcel Dekker, Inc., New York. 87.

48. Schwartz, M. L., S. V. Pizzo, R. L. Hill, and P. A. Mckee. 1973. Human factor XIII from plasma and platelets. Molecular weights, subunit structures, proteolytic activation and cross-linking of fibrinogen and fibrin. J. Biol. Chcm. 248: 1395. 